\title{
Cosegregation of two unlinked mutant alleles in some cases of autosomal dominant familial exudative vitreoretinopathy
}

\author{
BS Shastry*,1 and MT Trese ${ }^{2}$
}

\author{
${ }^{1}$ Department of Biological Sciences, Oakland University Rochester, MI 48309, USA; ${ }^{2}$ Department of Ophthalmology, \\ William Beaumont Hospital, Royal Oak, MI, USA
}

Familial exudative vitreoretinopathy (FEVR) is a bilateral, clinically and genetically heterogeneous hereditary eye disorder that affects both the retina and the vitreous body. The condition has a high degree of penetrance and variable expressivity. In some cases of autosomal dominant FEVR (AD FEVR), mutations in the frizzled-4 gene (FZD-4) have been shown to be involved in FEVR pathology. In this study, we report that a second unlinked gene (Factor V) is also mutated (Leiden mutation) in the same family, which harbors the FZD-4 gene mutation. These results show for the first time that some families with FEVR could be digenic. While this is unlikely to be a widespread problem, the occurrence of digenic disorders with apparently simple Mendelian inheritance patterns renders the current method of analysis of monogenic disorders by linkage and mutation screening incomplete.

European Journal of Human Genetics (2004) 12, 79-82. doi:10.1038/sj.ejhg.5201093

Published online 15 October 2003

Keywords: vitreoretinopathy; digenic; exudative; factor V; Leiden; mutation

\section{Introduction}

Familial exudative vitreoretinopathy (FEVR) is a hereditary eye disorder. It affects both the retina and the vitreous body. The condition is characterized by an abnormal vascularization of the peripheral retina, retinal folds, retinal exudates and tractional retinal detachment (Figure 1a). The disorder has a high degree of penetrance and variable expressivity. It is a bilateral disorder and systemic association is absent. ${ }^{1}$ Minimally affected individuals do not show any visual symptoms. Although the clinical manifestations of FEVR are similar to retinopathy of prematurity (ROP), FEVR patients do not have a history of oxygen therapy or low birth weight, and have a normal gestational period. In the majority of cases, FEVR is an autosomal dominant (AD) trait, ${ }^{2}$ but, recently, a series of $\mathrm{X}$-linked (XL) recessive ${ }^{3,4}$ and a few families with an

*Correspondence: Dr BS. Shastry. Tel: + 2483703577 ;

Fax: + 2483704225 ;

E-mail: shastry@oakland.edu

Received 20 May 2003; revised 19 July 2003; accepted 8 August 2003 autosomal recessive (AR) inheritance have been documented. ${ }^{5,6}$ While linkage analysis has not been reported for the $\mathrm{AR}$ form, one form of the $\mathrm{AD}$ and $\mathrm{XL}$ forms has been recently shown to be caused by segregating mutations in the frizzled-4 (FZD-4) and the Norrie disease (ND) gene, respectively. ${ }^{7-9}$ The FZD gene product can function as a receptor for Wnt proteins that have a major role in various cellular processes such as control of cell polarity, malignant transformation and determination of cell fate. Similarly, the ND gene product norrin may function as a dimeric growth factor in the retina. Additionally, the coagulation factor $\mathrm{V}$ Leiden mutation is reported to cause peripheral retinal neovascularization. Moreover, the $\mathrm{AD}$ and XL forms are genetically heterogeneous disorders. ${ }^{10,11}$

Materials and methods

In order to understand the relationship between genetic contribution and variable expressivity of FEVR pathology, we have studied six AD, four XL and four AR FEVR families 
(a total of 28 affected and 14 unaffected family members), with retinal findings consistent with FEVR disorder including the time of onset and tempo. However, with the exception of one $\mathrm{AD}$ and one XL family, none of the other families are large enough to allow linkage analysis. Members of the families underwent complete ophthalmological evaluation. A range of clinical manifestations has been seen in all of these families. Only one AD FEVR family (Figure 1b) in this group harbored a mutation in the FZD-4 gene. Since factor V Leiden mutation was reported to cause peripheral retinal neovascularization, we reasoned that it could be involved in some FEVR families that show a variable phenotype. For this purpose, genomic DNA was amplified with a pair of primers, using the procedure described previously. ${ }^{12}$ Briefly, the PCR conditions were 30 cycles of $1.5 \mathrm{~min}$ at $94^{\circ} \mathrm{C}, 1 \mathrm{~min}$ at $60^{\circ} \mathrm{C}, 1 \mathrm{~min}$ at $72^{\circ} \mathrm{C}$ in a buffer containing $5 \mathrm{U}$ of polymerase, $10 \mathrm{pmol}$ each of the
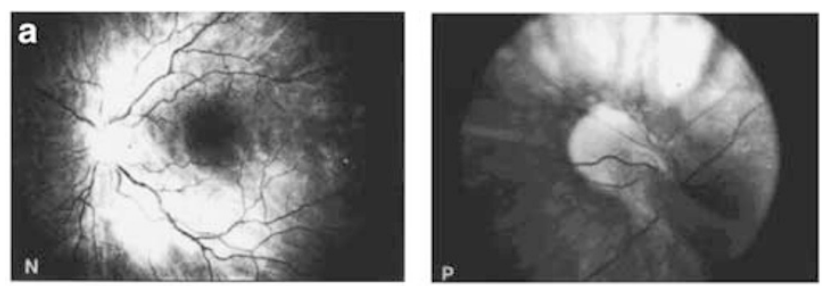

b

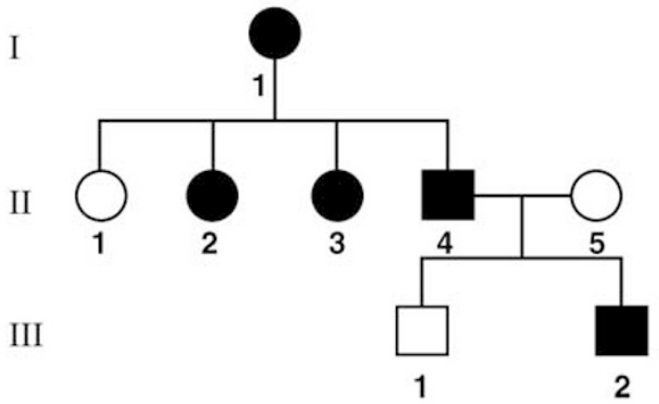

C

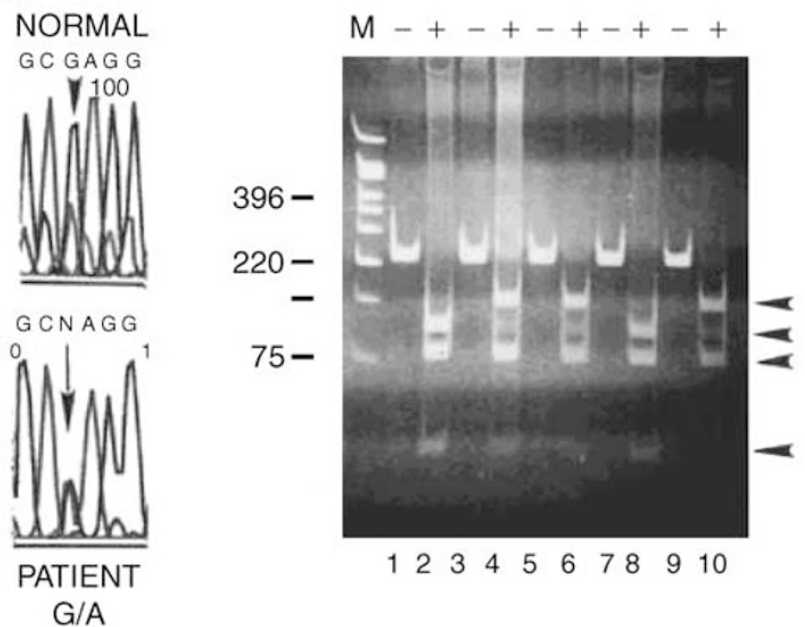

primer, $50 \mu \mathrm{M}$ each of the four doxynucleotides, $1.5 \mathrm{~mm}$ $\mathrm{MgCl}_{2}$ and $10 \mathrm{~mm}$ tris- $\mathrm{HCl} \mathrm{pH} \mathrm{8.3.} \mathrm{The} \mathrm{amplified} \mathrm{product}$ was subjected to restriction analysis. The base change was further identified by a big dye terminator cycle sequencing of the amplified product, according to the procedure supplied by the manufacturer (Applied Biosystems, Foster city, CA, USA). All parents were informed of the purpose of the study and it was approved by the Institutional Review Board of Oakland University, as well as by the Human Subject Investigation Committee of the William Beaumont Hospital.

\section{Results}

The coagulation factor $\mathrm{V}$ Leiden mutation was reported to cause peripheral retinal neovascularization and central retinal vein thrombosis. ${ }^{13,14}$ It is also weakly associated with advanced ROP, which is a phenocopy of FEVR. ${ }^{12}$ Additionally, in a small-scale study, the Leiden mutation is found to be present in 5\% of sporadic cases of FEVR. ${ }^{12}$ We therefore asked ourselves whether this mutation is also involved in familial FEVR pathology with a variable phenotype. Our extensive analysis has identified a heterozygous Leiden mutation in one familial case of AD FEVR (this same family has a segregating 2 base pair (bp) deletion mutation in the FZD-4 gene ${ }^{7}$ ). To detect the factor V Leiden mutation, genomic DNA was amplified with a pair of primers and the amplified product was subjected to

Figure 1 Mutational analysis of a digenic FEVR family. Fundus picture of a normal $(N)$ and an FEVR patient $(P)$ is shown in panel (a). The affected eye $(P)$ shows the retinal fold extending toward the retinal periphery and dragging of the optic disc. Panel (b) shows an autosomal dominant FEVR family, which harbors digenic mutation. The nucleotide sequence of the mutant part of exon 10 of the factor $V$ gene is shown in panel (c) (left part of the figure). The heterozygous sequence change in the patient is $G \rightarrow A$, which resulted in the amino acid glutamine instead of normal arginine, and destroyed one of the $M n / l$ sites. The right-hand side of panel (c) shows the restriction enzyme digestion pattern of PCR products to verify the sequence change. As expected, normal DNA (lanes 1 and 2, individual II-1 in the pedigree) after digestion with $\mathrm{MnIl}$ yielded 37, 82 and 104 bp fragments. The presence of 141, 104, 82 and 37 bp bands in lane 4 (patient I-1), lane 6 (patient II-4) and lane 10 (patient III-2) indicates a heterozygous mutation that is not present in the unaffected mother's DNA (lane 8, individual II-5) or other unrelated normal controls (data not shown). This mutation is perfectly segregating through three generations and with the disease. The same family has also shown a segregating 2 bp deletion in the FZD-4 gene. ${ }^{7} \mathrm{M}=$ marker lane, $(+)$ and ( - ) signs denote digested and undigested (input) DNA samples, respectively. The downward arrow in panel (c) shows the mutated nucleotide. 
restriction digestion using $10 \mathrm{U}$ of $\mathrm{Mnl}$ I enzyme (the Leiden mutation $\mathrm{G} \rightarrow \mathrm{A}$ in exon 10 of the factor $\mathrm{V}$ gene destroys one of the MnlI sites in the amplified fragment). The results are shown in Figure 1. As expected, the normal DNA (unaffected family member) after restriction digestion yields 37, 82 and $104 \mathrm{bp}$ fragments (Figure 1, panel c, lane 2). However, in the affected patients' DNA (three affected patients' samples are shown) the digestion produced 37, 82, 104 and $141 \mathrm{bp}$ fragments (lanes 4, 6 and 10), suggesting the loss of only one MnlI site (the presence of weaker 37 and $104 \mathrm{bp}$ bands is due to the heterozygosity of the mutation). DNA sequence analysis has further confirmed this base change as well as heterozygosity (panel c, left-hand side). Thus, all affected individuals harbored the same mutation and this mutation is absent in unaffected family members (panel c, lanes 2 and 8), as well as 40 normal controls from four different ethnic backgrounds (one of them matched to the family). The transition mutation, identified in the above study, converted the codon 506 arginine (R) to glutamine (Q) (CGA $\rightarrow$ CAA), which resulted in the loss of $\mathrm{MnlI}$ site. Mutational analysis of the remaining families did not reveal the Leiden mutation in the factor $\mathrm{V}$ gene as well as FZD-4 gene, but other mutations may be present in other exons or the promoter region of the gene. However, at present, it is not possible to determine the contributions of each gene to the phenotype because all affected individuals inherited both the mutations in the FZD-4 and factor V gene, and we do not have any additional individuals who have inherited mutations in only one of these genes.

\section{Discussion}

Owing to the highly variable pathology of FEVR even within a family, we hypothesized that an unlinked gene may be involved in some cases, similar to digenic retinitis pigmentosa. ${ }^{15}$ Strongly supporting evidence was obtained when we screened several families with FEVR pathology using a candidate gene approach. During our routine screening procedure, we have identified one AD FEVR family (Figure 1, panel b) that has digenic mutations. This same family was reported recently, and contains a segregating 2 bp deletion in the FZD-4 gene.

The above two genes that showed mutations in one FEVR family are not linked. The coagulation factor $\mathrm{V}$ is mapped to chromosome 1q21-25 and the FZD-4 gene to chromosome 11q. This cosegregation of unlinked genes in such a small family is statistically very unlikely, but there are precedents in the literature, for instance insulin resistance. ${ }^{16}$ Although the FEVR phenotype is highly variable among affected individuals within a family, one of the common features of the disorder in this family is the retinal detachment at an early age, which normally does not occur until the second decade of life. However, FZD-4 gene mutation alone in another family also shows a severe phenotype. $^{7}$ Thus, there is no direct correlation between the genotype and the phenotype in the present case. We do not know at present the mechanism by which digenic mutations contribute to the development of variable phenotypes of FEVR. The factor $\mathrm{V}$ Leiden mutation seems to enhance the risk of developing neovascular complication. ${ }^{14}$ Coagulation factor $\mathrm{V}$ undergoes proteolytic inactivation by activated protein $\mathrm{C}$ during normal hemostasis. The presence of arginine 506 in factor $\mathrm{V}$ was found to be essential for this proteolytic inactivation by activated protein $\mathrm{C}$. Thus, a mutation at this site must be producing a resistant factor $\mathrm{V}$ that may lead to abnormal hemostasis. Interestingly, a deficiency of protein $S$, which is a cofactor for activated protein $\mathrm{C}$, to inactivate factor $\mathrm{V}$, also produces an ROP type (a phenocopy of FEVR) clinical pathology. ${ }^{13}$ Additionally, it has been previously reported ${ }^{7}$ that mutant FZD-4 is defective in activating components of the Wnt/ $\mathrm{Ca}^{2+}$ signaling pathway (calcium/calmodulin-dependent protein kinase II and protein kinase C) due to its altered subcellular localization. Moreover, a mutation in mouse FZD-5 causes defective angiogenesis of the other tissue. ${ }^{17}$ Therefore, it is possible that these combined effects may contribute to the abnormal vascularization. However, it is not clear at present whether the Wnt signaling pathway meets with the anticoagulation pathway of factor $\mathrm{V}$ and how these combined defects lead to abnormal vascularization of the retina. Whatever the mechanism of the factor $\mathrm{V}$ gene mutation, identification of digenic mutations in an unlinked gene in an apparently simple Mendelian disease makes the current approach of linkage and mutational analysis of monogenic disorders incomplete and complicated, although such digenic disorders are rare. ${ }^{18}$

\section{Acknowledgements}

We would like to thank the families who provided the blood samples for this study. This study was supported in part by a grant from William Beaumont Hospital Research Institute (RI 02-01).

\section{References}

1 Shastry BS, Hiraoka M: Molecular genetics of familial exudative vitreoretinopathy and Norrie disease. Curr Genomics 2000; 1: 259-269.

2 Van Nouhuys CE: Dominant exudative vitreoretinopathy and other vascular developmental disorders of the peripheral retina. Doc Ophthalmol 1982; 54: 1-414.

3 Plager DA, Orgel IK, Ellis FD, Hartzer MK, Trese MT, Shastry BS: Xlinked recessive familial exudative vitreoretinopathy. Am J Ophthalmol 1992; 114: 145-148.

4 Fullwood P, Jones J, Bundey S, Dudgeon J, Fielder AR, Kilpatric MW: X-linked exudative vitreoretinopathy: clinical features and genetic linkage analysis. Br J Ophthalmol 1993; 77: 168-170.

5 Shastry BS, Trese MT: Familial exudative vitreoretinopathy: further evidence for genetic heterogeneity. Am J Med Genet 1997; 69: 217-218. 
6 De Crecchio G, Simonelli F, Mazzeo S et al: Autosomal recessive familial exudative vitreoretinopathy. Clin Genet 1998; 54: $315-320$.

7 Robitaile J, MacDonald MLE, Kaykas A et al: Mutant frzzled-4 disrupts retinal angiogenesis in familial exudative vitreoretinopathy. Nat Genet 2002; 32: 326-330.

8 Chen Z-Y, Bettinelli EM, Fielder A et al: A mutation in the Norrie disease gene associated with $\mathrm{X}$-linked familial exudative vitreoretinopathy. Nat Genet 1993; 5: 180-182.

9 Shastry BS, Hejtmancik JF, Plager DA, Hartzer MK, Trese MT: Linkage and candidate gene analysis of X-linked familial exudative vitreoretinopathy. Genomics 1995; 27: 341-344.

10 Shastry BS, Liu X, Hejtmancik JF, Plager DA, Trese MT: Evidence for genetic heterogeneity in X-linked familial exudative vitreoretinopathy. Genomics 1997; 44: 247-248.

11 Downey LM, Keen TJ, Roberts E, Mansfield DC, Bamashmus M, Inglehearn CF: A new locus for autosomal dominant familial exudative vitreoretinopathy maps to chromosome $11 \mathrm{p} 12-13$. Am J Hum Genet 2001; 68: 778-781.

12 Kleinberg S, Garrant S, Tillen T, Trese MT, Shastry BS: Factor V Leiden mutation (R506Q) and the risk of advanced retinopathy of prematurity (ROP). Int J Mol Med 2003; 12: 469-472.
13 Mintz-Hittner HA, Miyashiro MJ, Knignht-Nanan KM, O'Malley $\mathrm{RE}$, Marlar RA: Vitreoretinal findings similar to retinopathy of prematurity in infants with compound heterozygous protein S deficiency. Ophthalmology 1999; 106: 1525-1530.

14 Hvarfner C, Hillarp A, Larsson J: Influence of factor V Leiden on the development of neovascularization secondary to central retinal vein occlusion. Br J Ophthalmol 2003; 87: 305-306.

15 Kajawara K, Berson EL, Dryja TP: Digenic retinitis pigmentosa due to mutations at the unlinked peripherin/RDS and ROM 1 loci. Science 1994; 264: 1604-1608.

16 Savage DB, Agostini M, Barroso I et al: Digenic inheritance of severe insulin resistance in a human pedigree. Nat Genet 2002; 31: $379-384$.

17 Ishikawa T, Tamai Y, Zorn AM, Yoshida H, Seldin MF, Nishikawa S Mouse Wnt receptor gene FZD5 is essential for yolk sac and placental angiogenesis. Development 2001; 128: 25-33.

18 Ming JE, Muenke M: Multiple hits during early embryonic development: digenic diseases and holoprosencephaly. Am Hum Genet 2002; 71: 1017-1032. 